\title{
Linear inviscid wave propagation in a waveguide having a single boundary discontinuity: Part I: Theory
}

\author{
Charles Thompson \\ Department of Engineering Science and Mechanics, Virginia Polytechnic Institute and State University, \\ Blacksburg, Virginia 24061
}

(Received 18 March 1983; accepted for publication 5 September 1983)

\begin{abstract}
An examination of wave propagation in waveguides of rectangular cross section having a single boundary discontinuity is presented. Special attention is paid to waveguides with heights that are small compared to an acoustic wavelength. It is shown that the dynamic behavior of the enclosed fluid can be parametrized by the value of a single small parameter $\epsilon$, where $\epsilon$ is the ratio of the typical duct height $H_{0}$ to the wall wavelength $L_{0}$. The influence of planar discontinuities of zero and small but finite thickness on wave propagation is determined using the method of matched asymptotic expansions. Junction conditions, impedance across the junction, and uniformly valid composite expansions for the pressure in the duct are presented.
\end{abstract}

PACS numbers: 43.20.Mv, 43.20.Bi

\section{INTRODUCTION}

The topic which will be addressed in this paper concerns propagation of acoustic waves through two waveguides or cavities coupled through a junction discontinuity. We will use the method of matched asymptotic expansions, MMAE, to obtain an approximate solution for the oscillatory pressure. The enclosed fluid is taken to be inviscid and to respond linearly to harmonic excitation. In our analysis, we will examine waveguides which have rectangular cross section and have a height that varies as a function of horizontal position $X$ (see Fig. 1).

The types of boundary discontinuities that will be investigated fall into two categories, planar discontinuities of zero thickness and those which have small thickness when compared to an acoustic wavelength. We will limit our investigation to frequencies where the condition

$$
(\omega / c) H_{0} h\left(X / L_{0}\right)<1 \text {, }
$$

is satisfied. In this frequency range the fluid's behavior is parametrized by the ratio of two length scales $H_{0}$ and $L_{0} . H_{0}$ is the typical height of the smooth duct sections and $L_{0}$ represents the typical wall wavelength. We will also assume that

$$
\frac{\partial H(X)}{\partial X}=O\left(\frac{H_{0}}{L_{0}}\right)=O(\epsilon)<1,
$$

away from the discontinuity.

Some essential details of the present approach have been reported as early as 1897 by Rayleigh. ${ }^{1}$ Rayleigh, in his determination of the acoustic impedance of a thin aperture in an infinite baffle, made the argument that if the acoustic wavelength was long compared to the dimensions of the aperture, the fluid in proximity to the aperture behaves incompressibly. Hence, Rayleigh was first to utilize the relationship between physical dimensions of the aperture and the acoustic wavelength to analyze this problem in acoustic wave transmission. Tuck ${ }^{2}$ formalized Rayleigh's result using the method of matched asymptotic expansions.

Miles $^{3,4}$ and Schwinger et al..$^{5}$ using variational meth- ods determined the impedance across planar discontinuities of zero thickness in waveguides having otherwise uniform height. The impedance tabulated using these methods are extremely accurate. These results are insensitive to the choice made for the normal velocity distribution across the guide in the vicinity of the discontinuity. However, the pressure field is critically dependent on the choice of the velocity distribution. A poor choice can result in a slowly converging sum of weighted eigenfunctions of the uniform waveguides. Lesser and Lewis ${ }^{6}$ determined the acoustic impedance across a step discontinuity in an infinite two-dimensional waveguide with slowly varying height using MMAE. They showed that the acoustic impedance across the step discontinuity derived using the incompressible assumption is accurate to order $\epsilon^{2}$. However, they neglected to incorporate transcendentally small terms in the asymptotic behavior of the incompressible region. For infinite length waveguides these terms can be justifiably dropped. However, in a terminated guide these terms determine the critical distance between the termination and the discontinuity at which the interaction between the incompressible region and the wave region takes place.

In this paper we will present an analysis for long wavelength propagation in waveguides having a single boundary discontinuity. We will split the duct into three regions (see Fig. 2). In regions $A B$ and $C D$ the length scale which parametrizes the fluid's motion is the acoustic wavelength. Hence $A B$ and $C D$ will be called wave regions. In region $B C$ the parametrizing length scale is $H_{0}$. Since $H_{0}$ is small compared to an acoustic wavelength, $B C$ will be called the incompressible region. The problem will be analyzed using the method of matched asymptotic expansions MMAE.

Sections I and II will be devoted to the presentation and nondimensionalization of the linear inviscid equations which govern the wave and incompressible regions, respectively. The pressure and particle velocities in each region will be expressed in terms of a truncated asymptotic sequence in $\epsilon$. 
In Sec. III we will present the results of the matching process. Applying Van Dyke's' matching principle to the wave and incompressible solutions, several results will be obtained. We will show that continuity in volume velocity and pressure across the discontinuity is valid to first order in $\epsilon$. If the discontinuity has thickness, compressibility of the fluid must be taken into account at $O(\epsilon)$. We will show that interaction between the incompressible and wave region at $O\left(\epsilon^{2}\right)$ will occur if the distance between the discontinuity and duct termination, is $O\left[\epsilon \log \left(1 / \epsilon^{2}\right)\right]$. This distance is a multiplicative factor of $\log \left(1 / \epsilon^{2}\right)$ greater than the pressure boundary layer thickness, which is $O(\epsilon)$. The acoustic impedance across the discontinuity will be computed to $O\left(\epsilon^{3}\right)$. A uniform valid solution for the pressure valid to $O\left(\epsilon^{3}\right)$ will also be given.

\section{WAVE REGION}

In this section we will present a scheme for nondimensionalizing the linear inviscid equations of motion. The resulting nondimensional equations will govern acoustic wave propagation in the smooth sections of the duct.

The particle velocities $U^{*}, V^{*}$ and $W^{*}$ and pressure $P^{*}$ in the duct satisfy the following equations.

$$
\begin{aligned}
& \rho_{0} \frac{\partial U^{*}}{\partial T}+\frac{\partial P^{*}}{\partial X}=0 \\
& \rho_{0} \frac{\partial V^{*}}{\partial T}+\frac{\partial P^{*}}{\partial Y}=0 \\
& \rho_{0} \frac{\partial W^{*}}{\partial T}+\frac{\partial P^{*}}{\partial Z}=0
\end{aligned}
$$

and

$$
\frac{\partial U^{*}}{\partial X}+\frac{\partial V^{*}}{\partial Y}+\frac{\partial W^{*}}{\partial Z}+\frac{1}{\rho_{0} c^{2}} \frac{\partial P^{*}}{\partial T}=0,
$$

where $P^{*}$ is the oscillatory pressure, $\rho_{0}$ is the rest density of the fluid, $c$ is the speed of sound, and $U^{*}, V^{*}$, and $W^{*}$ represent the oscillatory particle velocities in the $x, y$, and $z$ directions, respectively.

Consider a rigid walled waveguide of rectangular cross section. The guide has an iris discontinuity in its upper wall which spans the guide's width along the $Z$ axis (see Fig. 1). If the fluid contained in the duct behaves in a linear and inviscid fashion, the only boundary condition that must be satisfied is the normal component of the velocity must equal zero on the boundary. We can write the boundary condition as $W^{*}(X, Y, Z=0)=0$,

$W^{*}\left(X, Y, Z=-L_{z}\right)=0$,

$V^{*}(X, Y=0, Z)=0$,

$V^{*}[X, Y=H(X), Z]=\frac{d H(X)}{d X} U^{*}[X, Y=H(X), Z]$

for $\mathrm{H}_{0} \delta^{-}>\mathrm{X}>\mathrm{H}_{0} \delta^{+}$,

and $\left(U^{*}, V^{*}\right) \cdot n=0$ on surface of the duct for $-H_{0} \delta^{-}<X \leqslant H_{0} \delta^{+}$. Since the duct is uniform in width, the pressure and particle velocity amplitudes can be expressed as the product of a function dependent on $Z$ alone and a func-

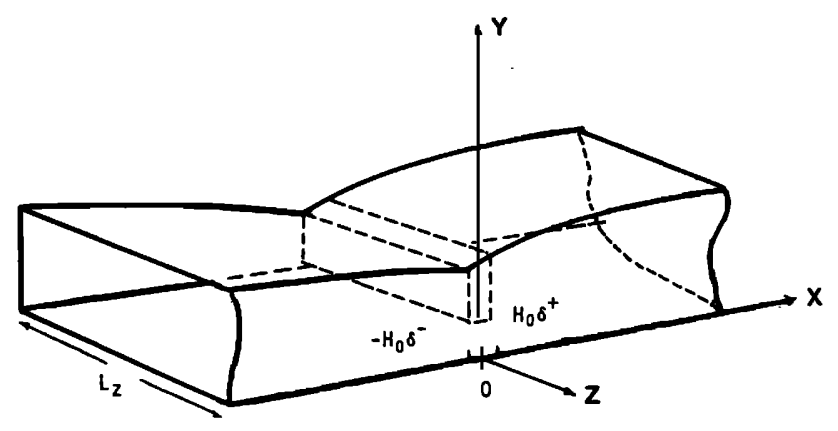

FIG. 1. Schematic diagram of a waveguide which has an iris discontinuity near the origin.

tion dependent on $X, Y$, and $T$.

$$
\begin{aligned}
& P^{*}(X, Y, Z, T)=G(Z) P(X, Y, T), \\
& U^{*}(X, Y, Z, T)=G(Z) U(X, Y, T), \\
& V^{*}(X, Y, Z, T)=G(Z) V(X, Y, T),
\end{aligned}
$$

and

$$
W^{*}(X, Y, Z, T)=-\frac{\partial G(Z)}{\partial Z} \int^{T} P d T
$$

where

$$
\frac{\partial G}{\partial Z}=0 \text { at. } Z=0 \text { and } Z=-L_{z} .
$$

Using the fact that $P *$ is a solution of the wave equation and the aforementioned boundary conditions in $Z$, it can be shown tht $G(Z)$ is equal to $\cos \left(m \pi Z / L_{z}\right)$. Because the solution is separable in $Z$ we need only solve the remaining twodimensional problem. Using Eq. (1) and dividing out $G(Z)$ in $P^{*}, U^{*}$, and $V^{*}$ yields

$$
\begin{aligned}
& \rho_{0} \frac{\partial U}{\partial T}+\frac{\partial P}{\partial X}=0, \\
& \rho_{0} \frac{\partial V}{\partial T}+\frac{\partial P}{\partial X}=0, \\
& \frac{\partial U}{\partial X}+\frac{\partial V}{\partial Y}+\frac{1}{\rho_{0} c^{2}} \frac{\partial P}{\partial T}+\tilde{\beta}_{m}^{2} \frac{1}{\rho_{0}} \int^{T} P d T=0,
\end{aligned}
$$

where $U, V$, and $P$ are functions of $X, Y$, and $T$ only and $\tilde{\beta}_{m}=m \pi / L_{z}$. To regain the three-dimensional solution we need only to multiply $U, V$, and $P$ by $G(Z)$. The particle velocity in the $Z$ direction is simply

$$
W=+\frac{\tilde{\beta}_{m} \sin \tilde{\beta}_{m} Z}{\rho_{0}} \int^{T} P d T
$$

Now let us examine the remaining two-dimensional problem. In Fig. 2 we see that the dynamic behavior of the enclosed fluid is characterized by the relationship between four length scales namely: (1) the acoustic wavelength $\lambda$, (2) the wall wavelength $L_{0},(3)$ the typical wall height $H_{0}$, and (4) the width of the guide in the $Z$ direction, $L_{z}$.

Let us first make some global assumptions about the relationship between the aforementioned length scales. We will assume that

$$
\text { and }
$$$$
L_{0} / \lambda=O(1), \quad L_{z} / \lambda=O(1),
$$

$$
H(X)=O\left(H_{0}\right)
$$



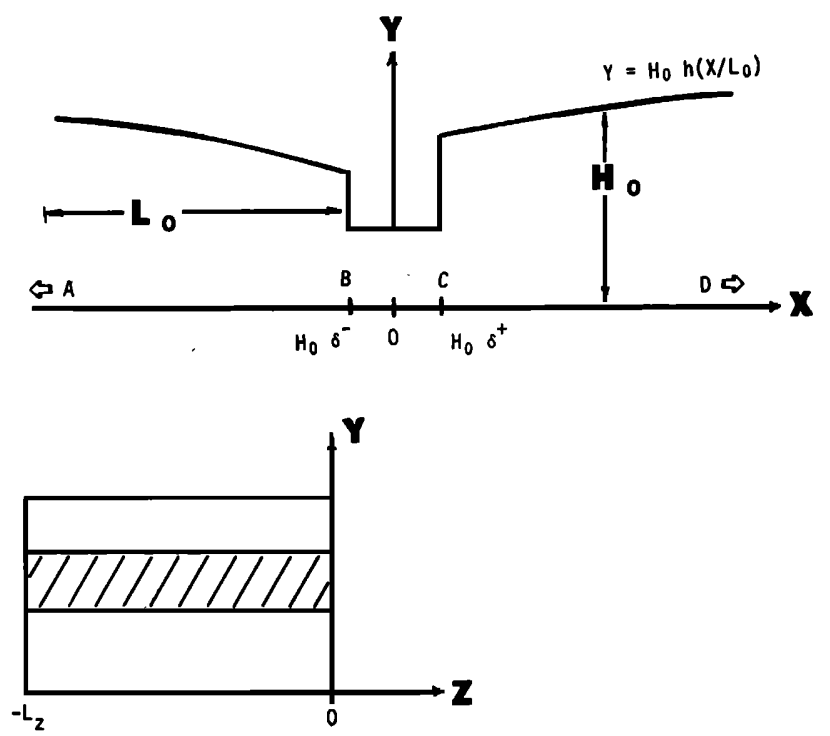

FIG. 2. This diagram denotes the four important length scales governing the dynamic behavior of the enclosed fluid. These length scales are (1) the acoustic wavelength $\lambda,(2)$ the wall wavelength $L_{0},(3)$ the typical duct height $H_{0}$, and (4) the duct width $L_{z}$.

in the duct. We will also assume no cross modes in $Y$ will propagate in the duct. Hence, the condition

$$
\left[(\omega / c) L_{0}\right]\left(H_{0} / L_{0}\right)<1,
$$

or equivalently

$$
\epsilon k<1 \text {, }
$$

where $\epsilon=H_{0} / L_{0}$ is a small parameter, and $k=(\omega / c) L_{0}$ is the nondimensional wavenumber is assumed to be satisfied.

In the regions $\mathrm{AB}$ and $\mathrm{CD}$, termed the wave regions, the height of the duct varies slowly with $X$. The length scale parametrized both wave and wall variation is $L_{0}$. The slope of the upper wall in the wave region is assumed to be of $O(\epsilon)$.

$$
\frac{d H(X)}{d X}=\frac{H_{0}}{L_{0}} \frac{d h\left(X / L_{0}\right)}{d\left(X / L_{0}\right)}=\epsilon h^{\prime}\left(\frac{X}{L_{0}}\right)=O(\epsilon),
$$

where $h^{\prime}=O(1)$.

The normal component of the oscillatory particle velocity equals zero on the upper and lower walls. Given that the duct slope is $O(\epsilon)$, we expect the typical vertical velocity, $V_{0}$ to be related to the horizontal velocity, $U_{0}$, as

$$
V_{0}=\epsilon U_{0} \text {. }
$$

Our nondimensionalization scheme for the oscillatory variables in the wave region is

$$
u=U / U_{0}, \quad v=V / V_{0}=V /\left(U_{0} \epsilon\right), \quad w=W / U_{0},
$$

and

$$
p=P /\left(\omega \rho_{0} L_{0} U_{0}\right) .
$$

For the coordinates the nondimensionalization is

$$
\begin{aligned}
& x^{+}=\left(X-\delta^{+} H_{0}\right) / L_{0}, \quad x^{-}=\left(X+\delta^{-} H_{0}\right) / L_{0}, \\
& y=Y / H_{0},
\end{aligned}
$$

and

$$
z=Z / L_{0}
$$

where

$$
t=\omega T, \quad k=\omega L_{0} / c, \quad \beta_{m}^{2}=\left(m \pi / L_{z}\right)^{2} L_{0}^{2},
$$

and

$$
g=\cos \beta_{m} z .
$$

Choosing the nondimensional time dependence to be $e^{-i t}$ the complex amplitudes of the particle velocities and pressure satisfy the equations

$$
\begin{array}{ll}
i u=p_{x^{+}}, & \epsilon^{2} i v=p_{y}, \\
i w=p g_{z}, & u_{x^{+}}+v_{y}=i \hat{k}^{2} p,
\end{array}
$$

for $x^{+}>0$, and

$$
\begin{array}{ll}
i u=p_{x^{-}}, & \epsilon^{2} i v=p_{y}, \\
i w=p g_{z}, & u_{x^{-}}+v_{y}=i \hat{k}^{2} p,
\end{array}
$$

for $x^{-}<0$, where

$$
\beta_{m}^{2}=\left(m \pi L_{0} / L_{z}\right)^{2}, \quad \hat{k}^{2}=k^{2}-\beta_{m}^{2},
$$

and

$$
g=\cos \beta_{m} 2 .
$$

The wall boundary conditions are

$$
\begin{aligned}
& v=0 \text { at } y=0, \\
& v=h^{\prime}\left(x^{+}+\epsilon \delta^{+}\right) u \text { at } y=h\left(x^{+}+\epsilon \delta^{+}\right) \text {for } x^{+}>0, \\
& v=h^{\prime}\left(x^{-}-\epsilon \delta^{-}\right) u \text { at } y=h\left(x^{-}-\epsilon \delta^{-}\right) \text {for } x^{-}<0,
\end{aligned}
$$

and $(u, v) \cdot \mathbf{n}=0$ on the surface of the discontinuity. We will introduce the following for $p, u, v$, and $w$.

$$
\begin{array}{ll}
p=p\left(x^{ \pm}, y, t\right), & u=u\left(x^{ \pm}, y, t\right), \\
v=v\left(x^{ \pm}, y, t\right), & W=w\left(x^{ \pm}, y, z, t\right),
\end{array}
$$

where the superscript, \pm , on $x$ represents the region of space under investigation. The plus sign will be used when examining region $C D$ and the minus sign will be used when examining region $\mathbf{A B}$.

We will express the complex amplitudes $p, u, v$, and $w$ in terms of an asymptotic sequence in integer powers of $\epsilon$. Successive approximations for the pressure and particle velocity can be determined by evaluating higher order coefficients of this sequence.

$$
(p, u, v, w)=E_{2}(p, u, v, w),
$$

where

$$
E_{N} f\left(x^{ \pm}, y, z, \hat{k}, \epsilon\right)=\sum_{n=1}^{N} f_{n}\left(x^{ \pm}, y, z, \hat{k}\right) \epsilon^{n}+O\left(\epsilon^{N+1}\right) .
$$

Substituting Eq. (7) into Eqs. (5a), (5b), and (6) and equating like terms in $\epsilon$ yields at $\epsilon^{0}$

$$
\begin{aligned}
& p_{0}=p_{0}\left(x^{ \pm}, \hat{k}\right), \quad u_{0}=-i p_{0}^{\prime}, \\
& v_{0}=-i y\left(h^{\prime} / h\right) p_{0}^{\prime}, \quad w_{0}=-i g_{z} p_{0},
\end{aligned}
$$

and

$$
\left(p_{0}^{\prime} h\right)^{\prime}+\hat{k}^{2} h p_{0}=0 .
$$

At $\epsilon^{1}$

$$
\begin{aligned}
& p_{1}=p_{1}\left(x^{ \pm}, \hat{k}\right), \quad u_{1}=-i p_{0}^{\prime}\left(\dot{x}^{ \pm}, \hat{k}\right), \\
& v_{1}=-i y\left(h^{\prime} / h\right) p_{1}^{\prime}, \quad w_{1}=-i g_{z} p_{1},
\end{aligned}
$$

and

$$
\left(p_{1}^{\prime} h\right)^{\prime}+\hat{k}^{2} h p_{1}=0
$$


At $\epsilon^{2}$

$$
\begin{aligned}
p_{2}= & f_{2}\left(x^{ \pm}, \hat{k}\right)+\left(y^{2} / 2\right)\left(h^{\prime} / h\right) p_{0}^{\prime}, \\
u_{2}= & -i\left\{f_{2}^{\prime}+\left(y^{2} / 2\right)\left[\left(h^{\prime} / h\right) p_{0}^{\prime}\right]^{\prime}\right\}, \\
v_{2}= & i \hat{k}^{2}\left\{y f_{2}-\left(y^{3} / 6\right)\left[\left(h^{\prime} / h\right) p_{0}^{\prime}\right]\right\} \\
& +i\left(y f_{2}^{\prime \prime}-\left(y^{3} / 6\right)\left[\left(h^{\prime} / h\right) p_{0}^{\prime}\right]^{\prime \prime}\right\}, \\
w_{2}= & -i g_{z} p_{2},
\end{aligned}
$$

and $\left(h F^{\prime}\right)^{\prime}+\hat{k}^{2}(h F)=\left[\left(h^{\prime 2} h / 2\right) p_{0}^{\prime}\right]^{\prime}$ where

$$
\begin{gathered}
f_{2}=F+\left(h h^{\prime} / 6\right) p_{0}^{\prime}, \\
h=h\left(x^{ \pm} \pm \epsilon \delta^{ \pm}\right),
\end{gathered}
$$

and

$$
()^{\prime}=\frac{d()}{d x^{ \pm}} .
$$

The zeroth and first-order pressure coefficients, $p_{0}$ and $p_{1}$, are both solutions of the Webster horn equation. The second-order pressure coefficient $p_{2}$, is determined by solving the inhomogeneous Webster horn equation.

\section{INCOMPRESSIBLE REGION}

Now let us direct our attention to the oscillatory motion of the fluid in proximity to the wall discontinuity located in region $\mathrm{BC}$. As $x^{+}$and $x^{-}$becomes of $O(\epsilon)$, the vertical particle velocity $v$ becomes of $O(1 / \epsilon)$. The increase in the magnitude of the vertical component of the particle velocity is the result of the zero normal velocity boundary condition on the surface of the discontinuity. This boundary condition can only be satisfied if the fluid in the $x y$ plane undergoes a transition from one- to two-dimensional motion near the discontinuity. Therefore, the equations of motion derived for the wave region are singular in the limit as $\left|x^{ \pm}\right| \rightarrow \epsilon$. Hence, a locally valid set of equations must be derived to describe the fluid motion near the discontinuity. The region of influence of the discontinuity is taken to be of $O(\epsilon)$ in $x^{ \pm}$. Since this region of space in the $x y$ plane occupied by the discontinuity is small compared to an acoustic wavelength, it will be termed the incompressible region.

When $x^{ \pm}$becomes of $O(\epsilon), \epsilon v$ becomes of $O(1)$. Using this information a set of local variables and equations governing the fluid's behavior in region $\mathrm{BC}$ can be derived. The local oscillatory variables are

and

$$
\hat{u}=u, \quad \hat{v}=v \epsilon, \quad \hat{w}=w,
$$

$$
\hat{p}=p \text {. }
$$

For the coordinates the nondimensionalization is

$$
\begin{aligned}
& \hat{x}=\frac{X}{H_{0}}=\frac{x^{+}+\delta^{+} \epsilon}{\epsilon}=\frac{x^{-}-\delta^{-} \epsilon}{\epsilon}, \\
& \hat{y}=y,
\end{aligned}
$$

and

$$
\hat{z}=z \text {, }
$$

where

and

$$
t=\omega T, \quad k=\left(\frac{\omega}{c}\right) L_{0}, \quad \beta_{m}^{2}=\left(\frac{m \pi}{L_{z}}\right)^{2} L_{0}^{2},
$$

$$
g=\cos \beta_{m} \hat{z} .
$$

Substituting the aforementioned variables into Eqs. (3) and (4) and assuming $e^{-i t}$ time dependence for $\hat{u}, \hat{v}, \hat{w}$, and $\hat{p}$ yields

$$
\epsilon i \hat{u}=\hat{p}_{\hat{x}}, \quad \epsilon i \hat{v}=\hat{p}_{\hat{y}}, \quad \hat{u}_{\hat{x}}+\hat{v}_{\hat{y}}=i \hat{k} 2 \epsilon \hat{p},
$$

and

$$
i \widehat{w}=\hat{p} g_{z},
$$

where

$$
\beta_{m}^{2}=\left(\frac{m \pi L_{0}}{L_{z}}\right)^{2}, \quad \hat{k}^{2}=k^{2}-\beta_{m}^{2},
$$

and

$$
g=\cos \beta_{m} z
$$

The variables $\hat{u}, \hat{v}, \hat{w}$, and $\hat{p}$ represent the complex amplitudes of the particle velocities and pressure. The boundary conditions on the walls are

$$
\begin{aligned}
& \hat{v}=\epsilon h^{\prime}\left( \pm \epsilon \delta^{ \pm}\right) \hat{u}+\epsilon^{2}\left(\hat{x} \pm \delta^{ \pm}\right) h^{\prime \prime}\left( \pm \epsilon \delta^{ \pm}\right) \hat{u} \\
& \text { at } \hat{y}=h(\epsilon \hat{x}) \text { for }-\delta^{-}>\hat{x}>\delta^{+},
\end{aligned}
$$

and

$$
(\hat{u}, \hat{v}) \cdot \mathbf{n}=\mathbf{0},
$$

on the surface of the discontinuity. The local complex amplitudes $\hat{u}, \hat{v}$, and $\hat{p}$ are dependent on the stretched coordinate $\hat{x}$, the vertical position $\hat{y}$, and nondimensional wavenumber $\hat{k}$. The pressure and velocity amplitude will be expressed as a truncated asymptotic sequence in $\epsilon$.

$$
(\hat{p}, \hat{u}, \hat{v}, \hat{w})=E_{2}(\hat{p}, \hat{u}, \hat{v}, \hat{w}),
$$

where

$$
E_{N} \hat{f}(\hat{x}, \hat{y}, \hat{z}, \hat{k}, \epsilon)=\sum_{n=0}^{N} \hat{f}_{n}(\hat{x}, \hat{y}, \hat{z}, \hat{k}) \epsilon^{n}+O\left(\epsilon^{N+1}\right) .
$$

Substituting Eq. (13) into (11) and equating like terms in $\epsilon$ yields at $\boldsymbol{\epsilon}^{\mathbf{0}}$

$$
\hat{u}_{0_{\bar{x}}}+\hat{v}_{0_{\hat{y}}}=0, \quad \hat{w}_{0}=-i g_{\hat{z}} \hat{p}_{0},
$$

and

$$
\hat{p}_{0_{\hat{x}}}=\hat{p}_{0_{\hat{y}}}=0 \text {, }
$$

with boundary conditions

$$
\begin{aligned}
& \hat{v}_{0}=0 \text { at } \hat{y}=0, \\
& \hat{v}_{0}=0 \text { at } \hat{y}=h(\epsilon \hat{x}) \text { for }-\delta^{-}>\hat{x}>\delta^{+},
\end{aligned}
$$

and the normal velocity equaling zero on the surface of the discontinuity. At $\epsilon^{1}$

$$
\begin{aligned}
& \hat{u}_{1_{\hat{x}}}+\hat{v}_{1_{\hat{y}}}=i \hat{k}^{2} \hat{p}_{0}, \quad \hat{u}_{0}=-i \hat{p}_{1_{\hat{x}}}, \\
& \hat{v}_{0}=-i \hat{p}_{1_{\hat{y}}}, \quad \hat{w}_{0}=-i g_{\hat{z}} \hat{p}_{1},
\end{aligned}
$$

and

$$
\hat{p}_{1_{\hat{x} x}}+\hat{p}_{1_{\hat{y} \hat{j}}}=0 \text {, }
$$

with boundary conditions

$$
\hat{v}_{1}=0 \text { at } \hat{y}=0,
$$

and

$$
\begin{aligned}
\hat{v}_{1} & =\hat{u} h^{\prime}\left( \pm \epsilon \delta^{ \pm}\right) \text {at } \hat{y} \\
& =h(\epsilon \hat{x}) \text { for }-\delta^{-}>\hat{x}>\delta^{+},
\end{aligned}
$$


and the normal velocity equaling zero on the discontinuity. At $\epsilon^{2}$

$$
\begin{aligned}
& \hat{u}_{2_{\varkappa}}+\hat{v}_{2_{\searrow}}=i \hat{k}^{2} \hat{p}_{1}, \quad \hat{u}_{1}=-i \hat{p}_{2_{\varkappa}}, \\
& \hat{v}_{1}=-i \hat{p}_{2_{\grave{y}}}, \quad \hat{w}_{1}=-i g_{2} \hat{p}_{1},
\end{aligned}
$$

and

$$
\hat{p}_{2_{\hat{s g}}}+\hat{p}_{\hat{2}_{\hat{y} \hat{j}}}=-\hat{k}^{2} \hat{p}_{0},
$$

with boundary conditions

$$
\begin{aligned}
& \hat{v}_{2}=0 \text { at } \hat{y}=0, \\
& \hat{v}_{2}=\hat{u}_{1} h^{\prime}\left( \pm \epsilon \delta^{ \pm}\right)+\left(\hat{x} \mp \delta^{ \pm}\right) \hat{u}_{0} h^{\prime \prime}\left( \pm \epsilon \delta^{ \pm}\right), \\
& \text {at } \hat{y}=h(\epsilon \hat{x}) \text { for }-\delta^{-}>\hat{x}>\delta^{+},
\end{aligned}
$$

and the normal velocity equaling zero on the discontinuity. Therefore, the pressure in the incompressible region is governed by the Laplace equation to second order in $\epsilon$ and the Poisson equation to third order in $\epsilon$.

\section{JUNCTION CONDITIONS AND COMPOSITE EXPANSIONS}

In the following segment we will perform the $\epsilon^{0}, \epsilon^{\mathbf{1}}$, and $\epsilon^{2}$ match. Careful attention will be given to intuition where appropriate. However, intuition is not always available. Hence, technique may prove to be the only guide for evaluating a uniformly valid solution. An attempt will be made to keep the discussion as integrated as possible by stating useful equations explicitly. The details of the formalism presented here can be found in the literature, ${ }^{7-9}$ and will not be incorporated into the body of the text.

\section{A. Zeroth-order match}

By the matching principle MP

$$
H_{0,0} p=\left(T E_{0} \hat{T} E_{0}-E_{0} T E_{0} \hat{T}\right) p=0,
$$

and

$$
H_{0,0} u=\left(T E_{0} \widehat{T} E_{0}-E_{0} T E_{0} \widehat{T}\right) u=0 .
$$

Let us first match the incompressible and wave solutions of the pressure at zeroth order in $\epsilon$. The first term of $H_{0,0} p$ is

$$
T E_{0} \widehat{T} E_{0} p=\lim _{x \pm \rightarrow 0^{ \pm}} p_{0}
$$

and the second term is

$$
E_{0} T E_{0} \widehat{T} p=\lim _{\hat{x} \rightarrow \pm \infty} \hat{p}_{0}
$$

Hence,

$$
\lim _{\hat{x} \rightarrow \pm \infty} \hat{p}_{0}=\lim _{x^{ \pm} \rightarrow 0^{ \pm}} p_{0} .
$$

The solution of $\hat{p}_{0_{x}}=\hat{p}_{0_{y}}=0$, from Eq. (14) is

$$
\hat{p}_{0}=\text { const, }
$$

for all $\hat{x}$. Using matching principle MP and the previous result yields

$$
\hat{p}_{0}=p_{0}\left(0^{+}\right)=p_{0}\left(0^{-}\right),
$$

which is the classical continuity in pressure condition. Likewise, $H_{0,0} u=0$ yields

$$
\lim _{\hat{x} \rightarrow \pm \infty} \hat{u}_{0}=\lim _{x^{ \pm} \rightarrow 0^{ \pm}} u_{0}
$$

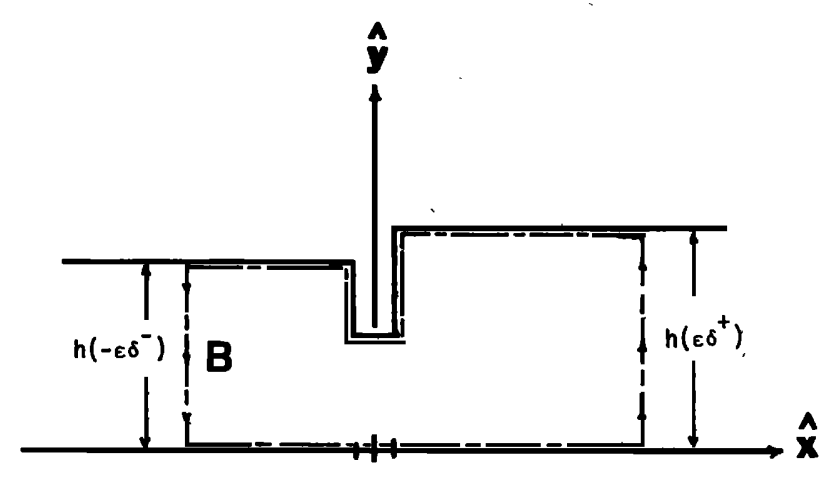

FIG. 3. Diagram showing the closed contour $B$ used to determine the junction condition obtained in Eq. (19).

Let us examine $\hat{u}_{0_{\dot{x}}}+\hat{v}_{0_{y}}=0$, also from Eq. (14). Applying the divergence theorem yields

$$
\int^{B} \hat{u}_{0} d \hat{y}-\hat{v}_{0} d \hat{x}=0
$$

assuming an outward normal to the boundary. In this twodimensional geometry, the contour $B$ traverses the boundary in a counterclockwise sense (see Fig. 3). Applying the boundary conditions and taking the outer limit aforementioned integral yields

$$
-\lim _{\hat{x} \rightarrow-\infty} \int_{0}^{h\left(-\epsilon \delta^{-}\right)} \hat{u}_{0} d \hat{y}+\lim _{\hat{x} \rightarrow+\infty} \int_{0}^{h\left(+\epsilon \delta^{+}\right)} \hat{u}_{0} d \hat{y}=O(\epsilon) .
$$

Interchanging the order of operation

$$
\begin{aligned}
& -\int_{0}^{h\left(-\epsilon \delta^{-}\right)} \lim _{\hat{x} \rightarrow-\infty} \hat{u}_{0} d \hat{y} \\
& +\int_{0}^{h\left(+\epsilon \delta^{+}\right)} \lim _{\hat{x} \rightarrow+\infty} \hat{u}_{0} d \hat{y}=O(\epsilon) .
\end{aligned}
$$

Since $\lim _{\hat{x} \rightarrow \pm \infty} \hat{u}_{0}=\lim _{x^{ \pm} \rightarrow 0^{ \pm}} u_{0}$, by the matching principle,

$$
h\left(-\epsilon \delta^{-}\right) u_{0}\left(0^{-}\right)=h\left(+\epsilon \delta^{+}\right) u_{0}\left(0^{+}\right)=q_{0}=\lim _{x \rightarrow \pm \infty} \hat{q}_{0},
$$

which is classical continuity in volume velocity condition. Therefore, the classical continuity conditions are valid to order $\epsilon$.

\section{B. First-order match}

We must evaluate the limiting conditions on the coefficients of $H_{1,0} p, H_{1,1} p$, and $H_{0,1} p$ when each is set equal to zero in order to complete the first-order match in pressure. By the matching principle

$$
H_{1,0} p=0 .
$$

Expanding $H_{1,0} p=0$ yields

$$
\left(T E_{1} \widehat{T} E_{0}-E_{0} T E_{1} \hat{T}\right) p=0 .
$$

Let us examine the first term in the above expression, $T E_{1} \widehat{T} E_{0} p . E_{0} p$ in the inner limit can be expressed in terms of a Taylor series in $p_{0}$ about $x^{ \pm}=0^{ \pm}$.

$T E_{1} \widehat{T} \lim _{x^{ \pm} \rightarrow 0^{ \pm}} E_{0} p=T E_{1} \widehat{T}\left[p_{0}\left(0^{ \pm}\right)+x^{ \pm} p_{0}^{\prime}\left(0^{ \pm}\right)+\cdots\right]$, 
$\widehat{T}$ transforms $x^{ \pm}$to $\epsilon\left(\hat{x} \mp \delta^{ \pm}\right)$and $E_{1}$ truncates the new series after the first term in $\epsilon$

$T\left(E_{1} \hat{T} \lim _{x^{ \pm} \rightarrow 0^{ \pm}} E_{0} p\right)=T\left[p_{0}\left(0^{ \pm}\right)+\epsilon\left(\hat{x} \mp \delta^{ \pm} \mid p_{0}^{\prime}\left(0^{ \pm}\right)\right]\right.$.

The final transformation $T$ converts $\hat{x}$ to $\left(x^{ \pm} \pm \epsilon \delta^{ \pm}\right) / \epsilon$. Hence,

$$
T E_{1} \hat{T} E_{0} p=p_{0}\left(0^{ \pm}\right)+x^{ \pm} p_{0}^{\prime}\left(0^{ \pm}\right) .
$$

The second term, $E_{0} \widehat{T} E_{1} T p$ is just $E_{0} T$ operating on the outer limit of the two first terms of the incompressible solution.

$$
E_{0} T \lim _{\hat{x} \rightarrow \pm \infty}\left(\hat{p}_{0}+\epsilon \hat{p}_{1}\right)=E_{0} T\left(E_{1} \widehat{T}_{p}\right),
$$

for the complete match the commutator matrix $H_{1,0} p$ must equal zero. Therefore,

$$
E_{0} T \lim _{\hat{x} \rightarrow \pm \infty}\left(\hat{p}_{0}+\epsilon \hat{p}_{1}\right)=p_{0}\left(0^{ \pm}\right)+x^{ \pm} p_{0}^{\prime}\left(0^{ \pm}\right) .
$$

It is easily seen that the above expression can be rewritten as

$$
\lim _{\hat{x} \rightarrow \pm \infty} \hat{p}_{1}=\left(\hat{x} \mp \delta^{ \pm}\right) p_{0}^{\prime}\left(0^{ \pm}\right),
$$

since $\lim _{\hat{x} \rightarrow \pm \infty} p_{0}$ is equal to $p_{0}\left(0^{ \pm}\right)$. Taking the outer limit of this expression and utilizing the fact that $\lim _{\hat{x} \rightarrow \pm \infty} \hat{u}_{0}=u_{0}\left(0^{ \pm}\right)$, then

$$
\lim _{\hat{x} \rightarrow \pm \infty} \hat{p}_{1}=i \hat{x} u_{0}\left(0^{ \pm}\right)
$$

where $i \hat{u}_{0}\left(0^{ \pm}\right)=p_{0}^{\prime}\left(0^{ \pm}\right)$.

Next let us consider the evaluation of the coefficients of $H_{1,1} p$. By our previous definition of the commutator matrix, $H_{1,1} p$ can be expanded as

$$
H_{1,1} p=\left(T E_{1} \widehat{T} E_{1}-E_{1} T E_{1} \widehat{T}\right) p=0 .
$$

The first term $T E_{1} \widehat{T} E_{1} p$ will be evaluated. $E_{1} p$ in the inner limit can be expressed in terms of a Taylor series in $p_{0}$ and $p_{1}$ about $x^{ \pm}=0^{ \pm}$. Hence,

$$
\begin{aligned}
T E_{1} \dot{\widehat{T}} \lim _{x \rightarrow \pm 0} E_{1} p= & T E_{1} \hat{T}\left[p_{0}\left(0^{ \pm}\right)+x^{ \pm} p_{0}^{\prime}\left(0^{ \pm}\right)+\cdots\right. \\
& +\epsilon p_{1}\left(0^{ \pm}\right)+\epsilon x^{ \pm} p_{1}^{\prime}\left(0^{ \pm}\right) \\
& \left.+\epsilon\left(x^{ \pm}\right)^{2} / 2 p_{1}^{\prime}\left(0^{ \pm}\right)+\cdots\right] .
\end{aligned}
$$

$E_{1} \widehat{T}$ transforms the bracketed term into

$$
T\left(E_{1} \widehat{T} E_{1} p\right)=T\left[p_{0}\left(0^{ \pm}\right)+\epsilon\left(\hat{x} \mp \delta^{ \pm}\right) p_{0}^{\prime}\left(0^{ \pm}\right)+\epsilon p_{1}\left(0^{ \pm}\right)\right],
$$

a truncated transformed version of the previous expression. Performing the transformation $T$ yields

$$
\left(T E_{1} \widehat{T} E_{1} p\right)=p_{0}\left(0^{ \pm}\right)+x^{ \pm} p_{0}^{\prime}\left(0^{ \pm}\right)+\epsilon p_{1}\left(0^{ \pm}\right) .
$$

The second term of the commutator matrix is the truncated incompressible solution operated on by the outer limit and $E_{1} T$. Hence, for a match

$$
E_{1} T \lim _{\hat{x} \rightarrow \pm \infty}\left(\hat{p}_{0}+\epsilon \hat{p}_{1}\right)=p_{0}\left(0^{ \pm}\right)+x^{ \pm} p_{0}^{\prime}\left(0^{ \pm}\right)+p_{1}\left(0^{ \pm}\right) .
$$

Since $\lim _{\hat{x} \rightarrow \pm \infty} \hat{p}_{0}$ and $p_{0}\left(0^{ \pm}\right)$are matched at zeroth order the limiting constraint is

$$
\lim _{\hat{x} \rightarrow \pm \infty} \hat{p}_{1}=\left(\hat{x} \mp \delta^{ \pm}\right) p_{0}^{\prime}\left(0^{ \pm}\right)+p_{1}\left(0^{ \pm}\right) \text {. }
$$

The evaluation of $H_{0,1} p$ is not necessary since it yields the redundant conclusion

$$
\lim _{\hat{x} \rightarrow \pm \infty} \hat{p}_{0}=p_{0}\left(0^{ \pm}\right)
$$

The effect of the pressure boundary layer has yet to be determined. It can be shown that

$$
\lim _{x \rightarrow \pm 0} p_{1}=\lim _{\hat{x} \rightarrow \pm \infty}\left[\hat{p}_{1}-\left(\hat{x} \mp \delta^{ \pm} \mid p_{0}\left(0^{ \pm}\right)\right]=p_{1}\left(0^{ \pm}\right),\right.
$$

is equivalent to the result given in Eq. (21). We must keep in mind that $p_{1}\left(0^{ \pm}\right)$is matched to within a constant. The value of this constant is governed by the terminal boundary conditions, i.e., boundary conditions at the ends of the guide.

The first-order pressure coefficient in the incompressible region, $\hat{p}_{1}$, is the solution of the Laplace Eq.(15). Applying the outer limit to $\hat{p}_{1}$ yields

$$
\begin{aligned}
\lim _{\hat{x} \rightarrow \pm \infty} \hat{p}_{1}= & \left(\hat{x} \mp \delta^{ \pm} \mid p_{0}^{\prime}\left(0^{ \pm}\right)+p_{1}\left(0^{ \pm}\right)\right. \\
& +\sum_{n=1}^{\infty} S_{n}^{ \pm} e^{-\left|\hat{x} \mp \delta^{ \pm}\right| \pi n / h\left( \pm \epsilon \delta^{ \pm}\right)} \\
& \times \cos \hat{y} n \pi / h\left( \pm \epsilon \delta^{ \pm}\right),
\end{aligned}
$$

where $S_{n}^{+}$, and $S_{n}^{-}$are scattering amplitudes. The values of $S_{n}^{+}$and $S_{n}^{-}$are determined by applying Lagrange's expansion theorem ${ }^{10}$ to $\hat{p}_{1}$. We can see that the aforementioned expression differs from that obtained in Eq. (21) by a summation of exponential terms. The contribution of this summation is exponentially small if the guide is infinite in extent. However, we must be cautious about neglecting such terms if the guide is terminated. For example, when the distance between the discontinuity and the guide termination is $O[\epsilon \log (1 / \epsilon)]$, the sum of exponential terms in Eq. (23) becomes sequence in powers of $\epsilon$. Therefore, reflection and retransmission of the first mode in $\hat{y}$ must be considered in the second-order match. If the distance between the termination and the discontinuity, $x_{T}^{ \pm}$, is

$$
\left|x_{\bar{T}}^{ \pm}\right| \geqslant \epsilon \log \left(1 / \epsilon^{3}\right) h( \pm \epsilon \delta) / \pi,
$$

the error introduced will be $O\left(\epsilon^{3}\right)$ in the outer limit. Since we are solving the problem to $O\left(\epsilon^{3}\right)$, the condition stated in Eq. (24) will be a useful indicator of when the first cross mode in $\hat{y}$ should be retained. If the termination is a distance of $O(\epsilon)$ away from the discontinuity, interaction between the pressure boundary layer and the wave solution will occur. Our approach as stated will fail. Assuming that Eqs. (24) and (22) hold, we can express the pressure drop across discontinuity as

$$
\begin{aligned}
p_{1}\left(0^{-}\right)-p_{1}\left(0^{+}\right)= & \lim _{\hat{x} \rightarrow-\infty}\left[\hat{p}_{1}-\left(\hat{x}+\delta^{-}\right) p_{0}^{\prime}\left(0^{-}\right)\right] \\
& -\lim _{\hat{x} \rightarrow \infty}\left[\hat{p}_{1}-\left(\hat{x}-\delta_{,}^{+}\right) p_{0}^{\prime}\left(0^{+}\right)\right] .
\end{aligned}
$$

Defining $\hat{p}_{1}$ in terms of the velocity potential $q_{0} \hat{\phi}_{1}$ yields

$$
\hat{p}_{1}=i q_{0} \hat{\phi}_{1}(\hat{x}, \hat{y}) \text {, }
$$

where $q_{0}$ is zeroth-order volume velocity evaluated at $x^{ \pm}=0^{ \pm}$. Using Eqs. (9) and (19), the pressure drop is 


$$
\begin{aligned}
\Delta p_{1}= & p_{1}\left(0^{-}\right)-p_{1}\left(0^{+}\right) \\
= & -i q_{0}\left(\lim _{\hat{x} \rightarrow+\infty}\left\{\hat{\phi}_{1}-\left[\left(\hat{x}-\delta^{+}\right) / h\left(+\epsilon \delta^{+}\right)\right]\right\}\right) \\
& -\lim _{\hat{x} \rightarrow-\infty}\left\{\hat{\phi}_{1}-\left[\left(\hat{x}+\delta^{-}\right) / h\left(-\epsilon \delta^{-}\right)\right]\right\} \\
= & -i q_{0} \Delta \hat{\phi}_{1} .
\end{aligned}
$$

Hence, the nondimensional acoustical impedance across the junction coupling the two smooth waveguides is

$$
\mathbf{Z}=\left(\Delta p_{0}+\epsilon \Delta p_{1} /\left(q_{0} / \hat{k}\right)=-\epsilon i \hat{k} \Delta \hat{\phi}_{1}+O\left(\epsilon^{2}\right)\right.
$$

where

$$
\Delta p_{0}=0,
$$

and

$$
\Delta p_{1}=p_{1}\left(0^{-}\right)-p_{1}\left(0^{+}\right)
$$

The discontinuity in the pressure in the wave region however, does not imply discontinuous behavior in the incompressible region, just that the asymptotes of the incompressible solution do not have the same value.

By the same matching technique it can be shown

$$
\lim _{\hat{x} \rightarrow \pm \infty} \hat{u}_{1}=u_{1}\left(0^{ \pm}\right)+\left(\hat{x} \mp \delta^{ \pm}\right) u_{0}^{\prime}\left(0^{ \pm}\right) \text {. }
$$

From Eq. (15)

$$
\hat{u}_{1_{x}}+\hat{v}_{1_{g}}=i \hat{k}^{2} \hat{p}_{0}
$$

Taking the surface integral of the above equation and then applying the divergence theorem to the result yields

$$
\int^{B}\left(\hat{u}_{1}, d \hat{y}-\hat{v}_{1} d \hat{x}\right)=\int^{S} i \hat{k}^{2} \hat{p}_{0} d S,
$$

assuming an outward normal to the boundary. The contour $B$ traverses the boundary in a counterclockwise sense and $S$ is the surface area in the $x y$ plane enclosed in $B$ (see Fig. 4). Applying the boundary conditions yields

$$
\begin{aligned}
\lim _{|\hat{x}| \rightarrow \infty} & {\left[h\left(+\epsilon \delta^{+}\right) \hat{u}_{1}(\hat{x}, \hat{y})-h\left(-\epsilon \delta^{-}\right) \hat{u}_{1}(-\hat{x}, \hat{y})+\hat{x} h^{\prime}\right.} \\
\times & \left(+\epsilon \delta^{+}\right) \hat{u}_{0}(\hat{x}, \hat{y})+\hat{x} h^{\prime}\left(-\epsilon \delta^{-} \mid \hat{u}_{0}(-\hat{x}, \hat{y})\right] \\
= & i k^{2} \hat{p}_{0} h\left(+\epsilon \delta^{+}\right) \hat{x}+i k^{2} \hat{p}_{0} h\left(-\epsilon \delta^{-}\right) \hat{x} \\
& +i \hat{k}^{2} \hat{p}_{0} S_{\delta}+O(\epsilon),
\end{aligned}
$$

where $S_{\delta}$ represents the surface area occupied by the fluid

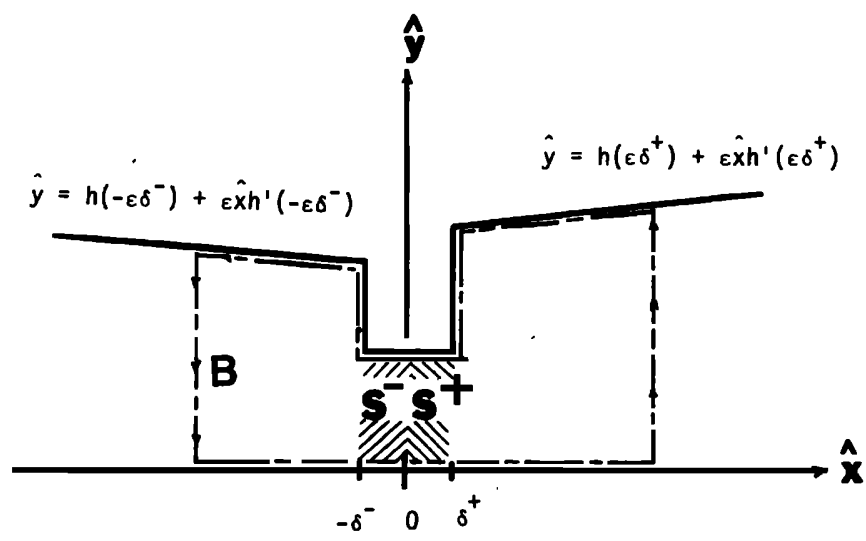

FIG. 4. Diagram showing the closed contour $B$ used to determine the junction condition obtained by Eq. (29).

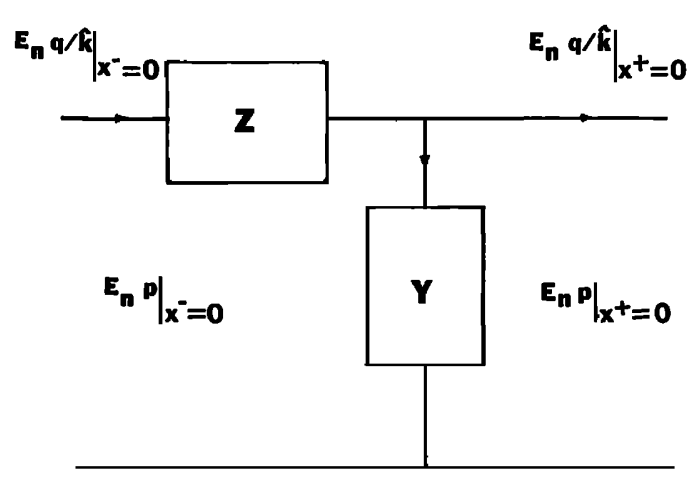

FIG. 5. Two port acoustic network representation of the discontinuity where $Z$ and $Y$ are defined as the nondimensional acoustic impedance and acoustic admittance across the junction, respectively.

beneath the discontinuity. Using the matching results given in Eqs. (28), (8), (18), and the boundary conditions, Eq. (28) can be rewritten as

$$
h\left(\epsilon \delta^{+}\right) u_{1}\left(0^{+}\right)=h\left(-\epsilon \delta^{-}\right) u_{1}\left(0^{-}\right)+i \hat{k}^{2} \hat{p}_{0} S_{\delta} .
$$

The nondimensional admittance to second order in $\epsilon$ across the junction is

$$
\mathbf{Y}=\left[\left(\Delta q_{0}+\epsilon \Delta q_{1}\right) / \hat{k}\right] / p_{0}\left(0^{-}\right)=-\epsilon i \hat{k} S_{\delta}+O_{0}\left(\epsilon^{2}\right)
$$

where

$$
\begin{aligned}
& \Delta q_{0}=0, \\
& \Delta q_{1}=-h\left(+\epsilon \delta^{+}\right) u_{1}\left(0^{+}\right)+h\left(-\epsilon \delta^{-}\right) u_{1}\left(0^{-}\right),
\end{aligned}
$$

and

$$
S_{\delta}=S^{+}+S^{-} \text {. }
$$

Note if the discontinuity has zero thickness $S_{\delta}$ equals zero and continuity volume holds to second order in $\epsilon$.

Using the junction impedance and admittance obtained Eqs. (26) and (30), the fluid's behavior near the discontinuity can model by a simple two-port acoustic network. In Fig. 5 we have schematically depicted a two-port representation of region BC. The impedance element $Z$ represents the effective acoustic mass of the fluid passing through the aperture. The first-order approximation for the value of this element is obtained solving the Laplace equation in region BC. The admittance element $\boldsymbol{Y}$ represents the acoustic compliance of the fluid in region BC. Recall that values for this model obtained matching the asymptotic behavior incompressible and wave solution for pressure and horizontal partial velocity in the limit as $\epsilon \rightarrow 0$. Hence this two-port representation of the fluid motion, for a fixed value of $\epsilon$, is only valid outside the incompressible region.

\section{Second-order match}

To complete the second-order match only $H_{2,1} p$ and $H_{2,2} p$ set equal to zero need to be computed. Since $H_{1,2} p$ set equal to zero yields a redundant result it will be disregarded here.

The coefficients of $H_{2,1} p=0$ are determined in the 
same fashion as in the previous sections. Matching $p$ and $\hat{p}$ via $H_{2,1} p=0$ yields

$$
\lim _{\hat{x} \rightarrow \pm \infty} \hat{p}_{2}=\left(\hat{x} \mp \delta^{ \pm}\right)^{2} / 2 p_{0}^{\prime \prime}\left(0^{ \pm}\right)+\left(\hat{x} \mp \delta^{ \pm}\right) p_{1}^{\prime}\left(0^{ \pm}\right) .
$$

Evaluating $H_{2,2} p=0$ yields the limiting condition that the

$$
\begin{aligned}
\lim _{x \rightarrow \pm \infty} \hat{p}_{2}= & p_{2}\left(0^{ \pm}, y\right)+\left(\hat{x} \mp \delta^{ \pm} \mid p_{1}^{\prime}\left(0^{ \pm}\right)\right. \\
& +\left(\hat{x} \mp \delta^{ \pm}\right)^{2} / 2 p_{0}^{\prime \prime}\left(0^{ \pm}\right) .
\end{aligned}
$$

The aforementioned result can be rewritten as

$$
\begin{gathered}
\lim _{\hat{x} \rightarrow \pm \infty}\left[\hat{p}_{2}(\hat{x}, \hat{y})-\left(\hat{x} \mp \delta^{ \pm}\right)^{2} / 2 p_{0}^{\prime \prime}( \pm 0)\right. \\
-\left(\hat{x} \mp \delta^{ \pm} \mid p_{1}^{\prime}\left(\left(0^{ \pm}\right)\right]=p_{2}\left(\left({ }^{ \pm}, y\right),\right.\right.
\end{gathered}
$$

where

$$
\begin{aligned}
p_{2}\left(0^{ \pm}\right)= & f_{2}\left(0^{ \pm}\right)+y^{2} / 2 \\
& \times\left[h^{\prime}\left( \pm \epsilon \delta^{ \pm}\right) / h\left( \pm \epsilon \delta^{ \pm}\right)\right] p_{0}^{\prime}\left( \pm \epsilon \delta^{ \pm}\right) .
\end{aligned}
$$

Rewriting Eq. (31) in terms of $f_{2}$ yields

$$
\begin{aligned}
\lim _{\hat{x} \rightarrow \pm \infty} & \left(\hat{p}_{2}(\hat{x}, \hat{y})-\left\{\left(\hat{x} \mp \delta^{ \pm}\right)^{2} / 2 p_{0}^{\prime \prime}\left(0^{ \pm}\right)\right.\right. \\
& \left.+\hat{y}^{2} / 2\left[h^{\prime}\left( \pm \epsilon \delta^{ \pm}\right) / h\left( \pm \epsilon \delta_{ \pm}\right)\right] p_{0}^{\prime}\left(0^{ \pm}\right)\right\} \\
& \left.-\left(\hat{x} \mp \delta^{ \pm}\right) p_{1}^{\prime}\left(0^{ \pm}\right)\right)=f_{2}\left(0^{ \pm}\right) .
\end{aligned}
$$

Recall that $\hat{p}_{2}$ satisfies the equation

$$
\hat{p}_{2_{\hat{x} \hat{x}}}+\hat{p}_{2_{p j}}=-\hat{k}^{2} \hat{p}_{0} \text {. }
$$

Looking at Eqs. (33), (34), and the boundary condition given in Eq. (15) we see that the second-order correction to the pressure $\hat{p}_{2}$ incorporates wall slope effects. To proceed further in our analysis we must determine the asymptotic behavior for $\hat{p}_{2}$ as $|\hat{x}|$ tends to infinity. We will represent $\hat{p}_{2}$ as the sum of two components, the first satisfying the Laplace equation and the boundary conditions outlined in Eq. (15) and the second satisfying Poission equation (34) and a homogeneous set of boundary conditions.

$$
\hat{p}_{2}=\hat{p}_{2}^{h}+\hat{p}_{2}^{p},
$$

where $p_{2}^{h}$ is the homogeneous solution of Eq. (34) and $p_{2}^{p}$ is the particular solution of Eq. (34). Let us outline the solution process for $\hat{p}_{2}^{h}$.

$$
\nabla^{2}\left(\hat{p}_{1}+\epsilon \hat{p}_{2}^{h}\right)=0,
$$

where

$$
\begin{aligned}
& \hat{v}_{0}+\epsilon \hat{v}_{1}=\epsilon \hat{u}_{1} h^{\prime}\left( \pm \epsilon \delta^{ \pm}\right) \text {at } y=h(\epsilon \hat{x}), \\
& \text { for }-\delta^{-}>\hat{x}>\delta^{+},
\end{aligned}
$$

and the normal velocity is equal to zero on the remaining portion boundary. By careful examination of the aforementioned boundary conditions we see that $\hat{p}_{2}^{h}$ is dependent on the solution to obtain for $\hat{p}_{1}$. Hence $\hat{p}_{2}^{h}$ actually represents high-order terms in the solution of $\hat{p}_{1}$. We will determine an expression for asymptotic behavior of $\hat{p}_{2}^{h}$ by conformal mapping the physical plane into the upper half of the $\omega$ plane (see Fig. 6). Mapping the $\hat{z}$ plane to the $\omega$ plane yields

$$
\frac{d \hat{z}}{d \omega}=\frac{d f(\omega)}{d \omega} \frac{\left(\omega-\omega_{B}\right)^{\gamma}}{\omega^{\gamma}}\left(\omega-\omega_{E}\right)^{\alpha},
$$
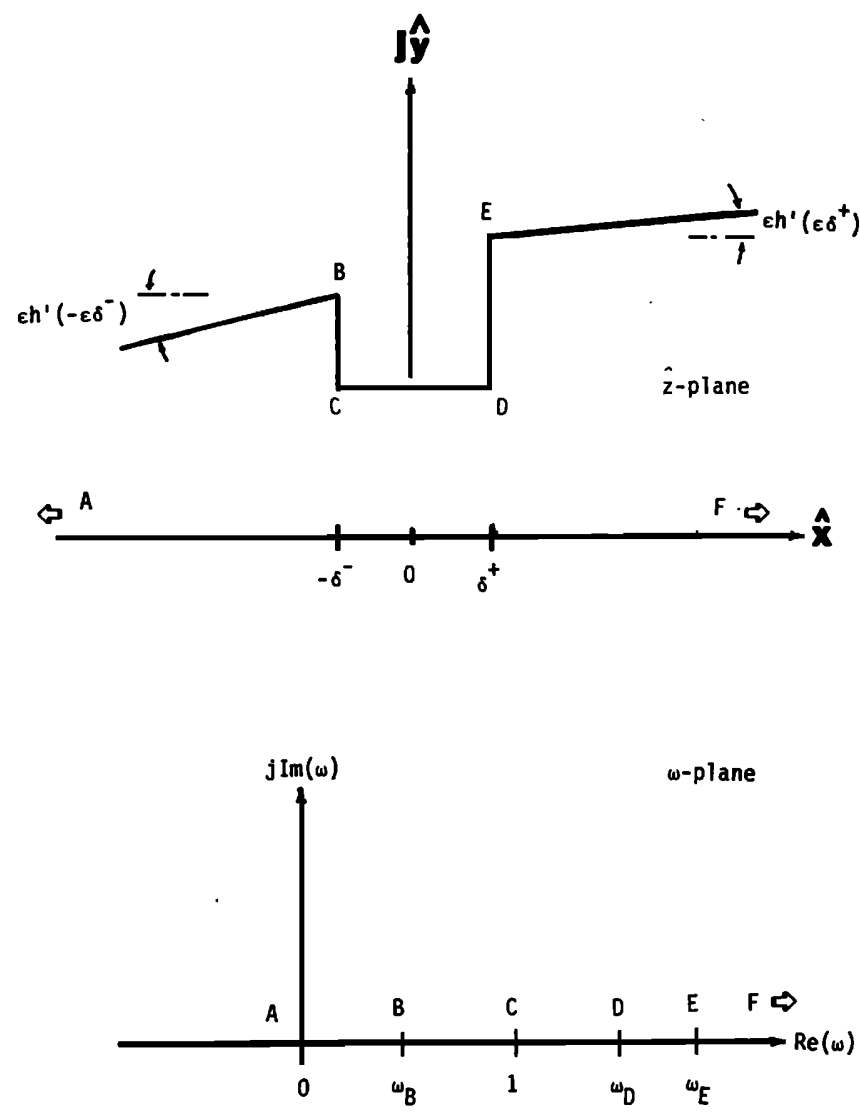

FIG. 6. Conformal map of the incompressible region.

where

$$
\begin{aligned}
& \hat{z}=\hat{x}+j \hat{y}, \\
& \frac{d f}{d \omega} \sim \frac{h\left(\epsilon \delta^{+}\right)}{\pi \omega} \text { as } \hat{x} \rightarrow+\infty \quad \text { or } \omega \rightarrow \infty, \\
& \frac{d f}{d \omega} \sim \frac{h\left(-\epsilon \delta^{-}\right)}{\pi \omega} \text { as } \hat{x} \rightarrow-\infty \text { or } \omega \rightarrow 0, \\
& \gamma=-\epsilon h^{\prime}\left(-\epsilon \delta^{-}\right) / \pi, \\
& \alpha=\epsilon h^{\prime}\left(\epsilon \delta^{+}\right) / \pi,
\end{aligned}
$$

and $d f / d \omega$ is the mapping function that would be obtained if the wall slope at $\hat{x}= \pm \infty$ were zero. Expanding $d \hat{z} / d \omega$ in a Taylor series about $\epsilon$ equal to zero yields

$$
\begin{aligned}
\frac{d \hat{z}}{d \omega} \approx & \frac{d f}{d \omega}\left[1+\gamma\left[\ln \left(\omega-\omega_{B}\right)-\ln \omega\right]\right. \\
& \left.+\alpha \ln \left(\omega-\omega_{E}\right)\right]+O\left(\epsilon^{2}\right),
\end{aligned}
$$

where

$$
\begin{aligned}
& \omega=\exp \left\{\pi\left[i q_{0} \Phi_{1}+i \epsilon q_{1}\left(0^{-}\right) \Phi_{2}^{h}\right] /\left[i q_{0}+i \epsilon q_{1}\left(0^{-}\right)\right]\right\}, \\
& \hat{p}_{1}=i \operatorname{Re}\left(q_{0} \Phi_{1}\right)=i q_{0} \hat{\phi}_{1},
\end{aligned}
$$

and

$$
\hat{p}_{2}^{h}=i \operatorname{Re}\left[q_{1}\left(0^{-}\right) \Phi_{2}^{h}\right]=i q_{1}\left(0^{-}\right) \hat{\phi}_{2}^{h} .
$$

Integrating the aforementioned expression and taking the limit $|\hat{x}|$ tend infinity yields 


$$
\begin{aligned}
\lim _{\hat{x \rightarrow \pm \infty}} \hat{p}_{2}^{h}= & i q_{1}\left(0^{-}\right)\left(\hat{\phi}_{1}-\frac{\left(\hat{x} \mp \delta^{ \pm}\right)}{h\left( \pm \epsilon \delta^{ \pm}\right)}\right)-p_{0}^{\prime}\left(0^{ \pm}\right) \frac{h^{\prime}\left( \pm \epsilon \delta^{ \pm}\right)}{h\left( \pm \epsilon \delta^{ \pm}\right)}\left(\frac{\left(\hat{x} \mp \delta^{ \pm}\right)^{2}-\hat{y}^{2}}{2}\right) \\
& +i q_{0}\left(\frac{h^{\prime}\left( \pm \epsilon \delta^{ \pm}\right)}{6}-\frac{h^{\prime}\left(\mp \epsilon \delta^{\mp}\right)}{\pi^{2}} \sum_{n=1}^{\infty} \frac{\theta^{n}}{n^{2}}\right)+O\left[\exp \left(-\frac{n \pi|\hat{x}|}{h\left( \pm \epsilon \delta^{ \pm}\right)}\right)\right],
\end{aligned}
$$

where

$$
\theta=\omega_{B} / \omega_{E}
$$

The asymptotic behavior of the particular solution of Eq. (34) is

$$
\lim _{\hat{x} \rightarrow \pm \infty} \hat{p}_{2}^{p}=-\hat{k}^{2} \hat{p}_{0}\left[\left(\hat{x} \mp \delta_{ \pm}\right)^{2} / 2\right]
$$

Using these asymptotic results we determine the second-order correction to pressure drop across the junction to be

$$
\begin{aligned}
\Delta f_{2}= & f_{2}\left(0^{-}\right)-f_{2}\left(0^{+}\right)=-i q_{1}\left(0^{-}\right)\left\{\lim _{\hat{x} \rightarrow \infty}\left[\hat{\phi}_{1}-\left(\hat{x}-\delta^{+}\right) / h\left(\epsilon \delta^{+}\right)\right]-\lim _{\ell \rightarrow-\infty}\left[\hat{\phi}_{1}-\left(\hat{x}+\delta^{-}\right) / h\left(-\epsilon \delta^{-}\right)\right]\right\} \\
& -\frac{i q_{0}}{3}\left[h^{\prime}\left(\epsilon \delta^{+}\right)-h^{\prime}\left(-\epsilon \delta^{-}\right)\right]-\frac{i q_{0}}{6}\left[h^{\prime}\left(\epsilon \delta^{+}\right)-h^{\prime}\left(-\epsilon \delta^{-}\right)\right]\left(\frac{1}{\pi^{2}} \sum_{n=1}^{\infty} \frac{\theta^{n}}{n^{2}}-1\right),
\end{aligned}
$$

or

$$
\Delta f_{2}=-i q_{1}\left(0^{-}\right) \Delta \hat{\phi}_{1}-i q_{0} \frac{\left[h^{\prime}\left(\epsilon \delta^{+}\right)-h^{\prime}\left(-\epsilon \delta^{-}\right)\right]}{3}-i q_{0}\left(\frac{h^{\prime}\left(\epsilon \delta^{+}\right)-h^{\prime}\left(-\epsilon \delta^{-}\right)}{6}\right)\left(\frac{1}{\pi^{2}} \sum_{n=1}^{\infty} \frac{\theta^{n}}{n^{2}}-1\right) .
$$

We will now tabulate impedance to third order in $\epsilon$. The nondimensional acoustic impedance across the junction is

$$
\begin{aligned}
\mathbf{Z}= & \frac{\epsilon \Delta p_{1}+\epsilon^{2} \Delta\left(p_{2}\right)}{\left[q_{0}+\epsilon q_{1}\left(0^{-}\right)\right] / \hat{k}}=-\epsilon i \hat{k} \Delta \phi_{1}-\epsilon^{2} i \hat{k}\left[\left(\frac{1}{3}\right)\left[h^{\prime}\left(\epsilon \delta^{+}\right)-h^{\prime}\left(-\epsilon \delta^{-}\right)\right]\right\} \\
& -\epsilon^{2} i \hat{k}\left(\frac{h^{\prime}\left(\epsilon \delta^{+}\right)-h^{\prime}\left(-\epsilon \delta^{-}\right)}{6}\right)\left(\frac{1}{\pi^{2}} \sum_{n=\phi}^{\infty} \frac{\theta^{2}}{n^{2}}-1\right)+O\left(\epsilon^{3}\right),
\end{aligned}
$$

where

$$
\Delta\left(p_{2}\right)=f_{2}\left(0^{-}\right)-f_{2}\left(0^{+}\right), \quad \theta=\omega_{B} / \omega_{E},
$$

and

$$
q_{1}\left(0^{-}\right)=h\left(-\epsilon \delta^{-}\right) u_{1}\left(0^{-}\right)
$$

As we can see, if $h^{\prime}\left(+\epsilon \delta^{+}\right)$equals $h^{\prime}\left(-\epsilon \delta^{-}\right)$or the discontinuity has zero thickness, the added mass reactance is zero and the impedance tabulated assuming the slope is zero is accurate to $O\left(\epsilon^{3}\right)$. Applying the match principle it can be shown that

$$
\lim _{\hat{x} \rightarrow \pm \infty} \hat{u}_{2}\left(0^{ \pm}, \hat{y}\right)=u_{2}\left(0^{ \pm}, y\right)+\left(\hat{x} \mp \delta^{ \pm}\right) \hat{u}_{1}^{\prime}\left(0^{ \pm}\right)+\left[\left(\hat{x} \mp \delta^{ \pm}\right)^{2} / 2\right] u_{0}^{\prime \prime}\left(0^{ \pm}\right)
$$

From Eq. (16)

$$
\hat{u}_{2_{\hat{x}}}+\hat{v}_{\hat{2}_{\hat{y}}}=i \hat{k}^{2} \hat{p}_{1}
$$

Taking the surface integral of the equation above and applying the divergence theorem to the result yields

$$
\int^{B}\left(\hat{u}_{2} d \hat{y}-\hat{v}_{2} d \hat{x}\right)=\int^{S} i \hat{k}^{2} \hat{p}_{1} d S,
$$

assuming the normal is directed out of the boundary (see Fig. 4). Applying the boundary condition in Eq. (16) and the matching results given in (21), (27), and (37) it can be shown that

$$
\begin{aligned}
\int_{0}^{h\left(+\epsilon \delta^{+}\right)} u_{2}^{+}(0, y) d y= & \int^{h\left(-\epsilon \delta^{-}\right)} \bar{u}_{2}(0, y) d y \\
& +i \hat{k}^{2}\left(\int^{S^{+}}\left[p_{1}\left(0^{+}\right)+\left.\left(h p_{0}\right)^{\prime}\right|_{x^{+}=0^{+}} \hat{x}\right] d S+\int^{S^{-}}\left[p_{1}\left(0^{-}\right)+\left.\left(h p_{0}\right)^{\prime}\right|_{x^{-}=0^{-}} \hat{x}\right] d S\right) .
\end{aligned}
$$

Therefore the shunt admittance to third order in $\epsilon$ is

$$
\begin{aligned}
\mathbf{Y}=\frac{\left(\Delta q_{0}+\epsilon \Delta q_{1}+\epsilon^{2} \Delta q_{2}\right) / \hat{k}}{p_{0}+\epsilon p_{1}\left(0^{+}\right)}= & -i \epsilon \hat{k}\left[S_{\delta}+\epsilon\left(S^{+} p_{1}\left(0^{+}\right)+S^{-} p_{1}\left(0^{-}\right)\right.\right. \\
& \left.\left.+\left.\frac{\left(h p_{0}\right)^{\prime}}{2}\right|_{x^{+}=0^{+}} \delta^{+} S^{+}-\left.\frac{\left(h p_{0}\right)^{\prime}}{2}\right|_{x^{-}=0^{-}} \delta^{-} S^{-}\right)\right]+O\left(\epsilon^{3}\right),
\end{aligned}
$$

where 


$$
\Delta q_{0}=0, \quad \Delta q_{1}=-h\left(+\epsilon \delta^{+}\right) u_{1}\left(0^{+}\right)+h\left(-\epsilon \delta^{-}\right) u_{1}\left(0^{-}\right),
$$

and

$$
\Delta q_{2}=-\int_{0}^{h\left(\epsilon \delta^{+}\right)} u_{2}\left(0^{+}, y\right) d y+\int_{0}^{h\left(-\epsilon \delta^{-}\right)} u_{2}\left(0^{-}, y\right) d y .
$$

\section{COMPOSITE EXPANSION}

Once the matching conditions are satisfied, the composite expansions for the pressure can be determined. The zerothorder composite solution is

$$
E_{0,0} p=p_{0}\left(x^{+}, \hat{k}\right)-p_{0}\left(0^{+}, \hat{k}\right)+O(\epsilon) \text { for } x^{+}+\epsilon \delta^{+} \geqslant 0,
$$

and

$$
E_{0,0} p=p_{0}(x, \hat{k})-p_{0}\left(0^{-}, \hat{k}\right)+O(\epsilon) \text { for } x^{-}-\epsilon \delta^{-} \leqslant 0 .
$$

The first-order composite solution is

$$
E_{1,1} p=p_{0}\left(x^{+}, \hat{k}\right)+\epsilon\left[p_{1}\left(x^{+}, \hat{k}\right)+\hat{p}_{1}(\hat{x}, \hat{y})\right]-\epsilon i q_{0}\left[\lim _{\hat{x} \rightarrow \infty} \hat{\phi}_{1}-\left(\hat{x}-\delta^{+}\right) / h\left(\epsilon \delta^{+}\right)\right]+\epsilon C_{1}+O\left(\epsilon^{2}\right) \text { for } x^{+}+\epsilon \delta^{+} \geqslant 0,
$$

and

$$
E_{1,1} p=p_{0}\left(x^{-}, \hat{k}\right)+\epsilon\left[p_{1}\left(x^{-}, \hat{k}\right)+\hat{p}_{1}(\hat{x}, \hat{y})\right]-\epsilon i q_{0}\left[\lim _{\hat{x} \rightarrow-\infty} \hat{\phi}_{1}-\left(\hat{x}+\delta^{-}\right) / h\left(-\epsilon \delta^{-}\right)\right]+\epsilon C_{1}+O\left(\epsilon^{2}\right)
$$

for $x^{-}-\epsilon \delta^{-} \leqslant 0$,

where the constant $C_{1}$ is determined by applying the terminal boundary conditions. The second-order composite solution is

$$
\begin{aligned}
E_{2,2} p= & p_{0}\left(x^{+}, \hat{k}\right)+\epsilon\left[p_{1}\left(x^{+}, \hat{k}\right)+\hat{p}_{1}(\hat{x}, \hat{y})\right]+\epsilon^{2}\left[p_{2}\left(x^{+}, y, \hat{k}\right)+\hat{p}_{2}(\hat{x}, \hat{y}, \hat{k})\right]-\left\{\lim _{x \rightarrow \infty}\left[\hat{\phi}_{1}-\left(\hat{x}-\delta^{+}\right) / h\left(+\epsilon \delta^{+}\right)\right]\right. \\
& \left.\times\left[i q_{0} \epsilon+i q_{1}\left(0^{-}\right) \epsilon^{2}\right]\right\}-\epsilon^{2}\left(f_{2}\left(0^{+}\right)+\frac{y^{2}}{2} \frac{h^{\prime}\left(+\epsilon \delta^{+}\right)}{h\left(+\epsilon \delta^{+}\right)} p_{0}^{\prime}\left(0^{+}\right)\right)+C_{1} \epsilon+C_{2} \epsilon^{2} \text { for } x^{+}+\epsilon \delta^{+} \geqslant 0,
\end{aligned}
$$

and

$$
\begin{aligned}
E_{2,2} p= & p_{0}\left(x^{-}, \hat{k}\right)+\epsilon\left[p_{1}\left(x^{-}, \hat{k}\right)+\hat{p}_{1}(\hat{x}, \hat{y})\right]+\epsilon^{2}\left[p_{2}\left(x^{-}, y, \hat{k}\right)+\hat{p}_{2}(\hat{x}, \hat{y}, \hat{k})\right]-\left\{\lim _{\hat{x} \rightarrow-\infty}\left[\hat{\phi}_{1}-\left(\hat{x}+\delta^{-}\right) / h\left(-\epsilon \delta^{-}\right)\right]\right. \\
& \left.\times\left[i q_{0} \epsilon+i q_{1}\left(0^{-}\right) \epsilon^{2}\right]\right\}-\epsilon^{2}\left(f_{2}\left(0^{-}\right)+\frac{y^{2}}{2} \frac{h^{\prime}\left(-\epsilon \delta^{-}\right)}{h\left(-\epsilon \delta^{-}\right)} p_{0}^{\prime}\left(0^{-}\right)\right)+C_{1} \epsilon+C_{2} \epsilon^{2} \text { for } x^{-}-\epsilon \delta^{-} \leqslant 0,
\end{aligned}
$$

where $C_{2}$ is determined from the terminal boundary conditions.

\section{CONCLUSION}

An analysis of long wavelength acoustic propagation in a waveguide having a single boundary discontinuity has been presented. Junction conditions as well as a uniformly valid expansion for the pressure have been determined. The junction impedances have been derived using the local solutions for the pressure near the discontinuity. The analysis of a number of problems using the results presented here have been completed ${ }^{12}$ and will be presented in a companion paper.

\section{ACKNOWLEDGMENT}

The author is indebted to Professor Patrick Leehey of the Massachusetts Institute of Technology for valuable suggestions on the earlier version of this manuscript. The author would also like to acknowledge C. Christie and J. MacKay for their assistance in the preparation of the manuscript.

'Lord Rayleigh, Philos. Mag. 44, 28 (1897).

${ }^{2}$ E. O. Tuck, J. Fluid Mech. 49, 65 (1971).

${ }^{3}$ J. W. Miles, J. Acoust. Soc. Am. 17, 259 (1946).

${ }^{4} J$. W. Miles, J. Acoust. Soc. Am. 17, 272 (1946).

5. Schwinger and D. S. Saxon, Discontinuities in Waveguides (Gordon and Breach, New York, 1968).

${ }^{6}$ M. B. Lesser and J. A. Lewis, J. Acoust. Soc. Am. 51, 1664 (1972).

${ }^{7}$ M. Van Dyke, Perturbation Methods in Fluid Mechanics (Parabolic, Stanford, 1964).

${ }^{8}$ L. E. Fraenkel, Proc. Cambridge Phil. Soc. 65, 209 (1969).

${ }^{9}$ W. Eckhaus, Matched Asymptotic Expansions and Singular Perturbations (North Holland, Amsterdam, 1973).

${ }^{10}$ Whitaker and Watson, Modern Analysis (Cambridge U. P., New York, 1927).

${ }^{11}$ M. B. Lesser and D. G. Crighton, Phys. Acoust. 10, 69 (1974).

${ }^{12}$ C. Thompson, J. Acoust. Soc. Am. 74, 356 (1983). 